\title{
The Semigeostrophic Equations Discretized in reference and dual variables
}

\author{
M. Cullen, W.Gangbo, G. Pisante
}

\begin{abstract}
We study the evolution of a system of $n$ particles $\left\{\left(x_{i}, v_{i}\right)\right\}_{i=1}^{n}$ in $\mathbb{R}^{2 d}$. That system is a conservative system with a Hamiltonian of the form $H[\mu]=$ $W_{2}^{2}\left(\mu, \nu^{n}\right)$, where $W_{2}$ is the Wasserstein distance and $\mu$ is a discrete measure concentrated on the set $\left\{\left(x_{i}, v_{i}\right)\right\}_{i=1}^{n}$. Typically, $\mu(0)$ is a discrete measure approximating an initial $L^{\infty}$ density and can be chosen randomly. When $d=1$, our results prove convergence of the discrete system to a variant of the semigeostrophic equations. We obtain that the limiting densities are absolutely continuous with respect to Lebesgue measure. When $\left\{\nu^{n}\right\}_{n=1}^{\infty}$ converges to a measure concentrated on a special $d$-dimensional sets, we obtain the Vlasov-Monge-Ampère (VMA) system. When, $d=1$ the VMA system coincides with the standard Vlasov-Poisson system.
\end{abstract}

Key words. mass transfer- Wasserstein metric AMS code: 49J40 82C40 47J25

\section{Introduction}

In this paper, we fix $n$ distinct points (centers) $c_{1}^{n}, \cdots, c_{n}^{n} \in \mathbb{R}^{2 d}$ and consider the Hamiltonians $H^{n}: \mathbb{R}^{2 n d} \rightarrow \mathbb{R}$ defined by

$$
H^{n}(\mathbf{z})=H^{n}\left(z_{1}, \cdots, z_{n}\right)=\frac{1}{2 n} \min _{\sigma \in S_{n}}\left\|\mathbf{z}-\mathbf{c}^{n, \sigma}\right\|^{2}
$$

where, $S_{n}$ is the set of permutations of $n$ letters, and

$$
\mathbf{c}^{n, \sigma}=\left(c_{\sigma(1)}^{n}, \cdots, c_{\sigma(n)}^{n}\right), \quad\left\|\mathbf{z}-\mathbf{c}^{n, \sigma}\right\|^{2}=\sum_{i=1}^{n}\left|z_{i}-c_{i}^{n, \sigma}\right|^{2} .
$$


Here, $|\cdot|$ denotes the Euclidean norm. One can readily check that $H^{n}$ is semiconcave since the eigenvalues of its second derivatives are less than or equal to $1 / n$. Thus, the set where it is not differentiable is $(2 n d-1)-$ rectifiable. For $\mathbf{z}=\left(z_{1}, \cdots, z_{n}\right)$, a point of differentiability of $H^{n}$, there exists a unique $\sigma_{\mathbf{z}} \in S_{n}$ which minimizes the expression in (1) ; in that case,

$$
n \nabla_{\mathbf{z}} H^{n}(\mathbf{z})=\mathbf{z}-\mathbf{c}^{n, \sigma_{\mathbf{z}}} .
$$

The Hamiltonian systems associated to $n H^{n}$ are then

$$
\left\{\begin{array}{l}
\dot{x}_{i}=v_{i}-b_{i}^{n, \sigma_{\mathbf{z}}} \\
\dot{v}_{i}=-\left(x_{i}-a_{i}^{n, \sigma_{\mathbf{z}}}\right) .
\end{array}\right.
$$

Here, we have used the notation $z_{i}=\left(x_{i}, v_{i}\right), c_{i}^{n}=\left(a_{i}^{n}, b_{i}^{n}\right) \in \mathbb{R}^{d} \times \mathbb{R}^{d}=$ $\mathbb{R}^{2 d}$. We study the Hamiltonian systems in (2) and their limits when $n$ tends to $+\infty$. We assume throughout this study that there exists a constant $E>0$ independent of $n$ such that

$$
\left|c_{i}^{n}\right| \leq E \quad \forall i=1, \cdots, n, \quad n=1,2, \cdots .
$$

Depending on the dimension $d$ and the geometry of the set $\left\{c_{i}^{n}\right\}_{i=1}^{n}$ when $n$ is large, the system in (2) converges to the 1 -dimensional VlasovPoisson system, the $d$-dimensional Vlasov-Monge-Ampère system or the semigeostrophic equations. The Vlasov-Poisson system appears in the fluids mechanics literature and has been extensively studied recently, [4], [7], [21], [22], in contexts completely different from ours. The Vlasov-MongeAmpère system was apparently discovered by Brenier [8] who considered the discrete system in (2) and its connection with the Euler incompressible equations as $n$ tends to $+\infty$. We also refer the reader to [10] and [20] for further studies. The semigeostrophic system was introduced as a model for large-scale flows of the atmosphere and ocean by Eliassen, [16], and Hoskins [19]. A semi-discrete solution procedure for them was introduced by Cullen and Purser, [14], [15]. Our discrete scheme is different from theirs. The continuous semigeostrophic system has been analysed by [9] and [13]. The Hamiltonian form of the continuous evolution equation was analysed by [23] and a discrete form related to ours was introduced by [6], whose study can be viewed as a preliminary to the current study. For wider reviews see also the monograph by L.C. Evans [17] and the book by Cullen, [12]. The relation of the Hamiltonian form of the semigeostrophic equations to that of the $2 \mathrm{~d}$ incompressible Euler equations is discussed by [23]. Discrete schemes for the $2 \mathrm{~d}$ incompressible Euler equations are reviewed by [3]. The convergence of the discrete scheme in (2) appears to be easier than the discrete scheme for the $2 \mathrm{~d}$ incompressible Euler equations as well as the one for the $n$-body problem because the velocity field is more regular.

We first collect useful notation which will be used throughout this paper and recall the definition of the Wasserstein distance between two probability measures defined on a normed space. 
We denote by $I_{d}$ the $d \times d$ identity matrix and by id $_{d}$ the identity map on $\mathbb{R}^{d}$. We denote by $I$ the $(2 d) \times(2 d)$ matrix and by id the identity map on $\mathbb{R}^{2 d}$. We denote by $J$ the symplectic matrix

$$
J=\left(\begin{array}{cc}
0 & -I_{d} \\
I_{d} & 0
\end{array}\right)
$$

which is the rotation of angle $\pi / 2$ when $d=1$.

- If $R>0$ and $z \in \mathbb{R}^{D}, B_{R}(z)$ denotes the ball in $\mathbb{R}^{D}$ of center $z$ and radius $R$. If $B \subset \mathbb{R}^{D}$ we denote by $B^{c}$ the complement of $B$.

- We denote by $\mathcal{P}(A)$ the set of Borel probability measures on a metric space ( $A$, dist). If $r>0$ and $\mu \in \mathcal{P}(A)$, the $r$-moment of $\mu$ with respect to the $x_{o} \in A$ is

$$
M_{r}(\mu)\left(x_{o}\right)=\int_{A} \operatorname{dist}^{r}\left(x, x_{o}\right) d \mu(x) .
$$

When $M_{2}(\mu)(0)<+\infty$, we write $\mu \in \mathcal{P}_{2}(A)$. In case $A \subset \mathbb{R}^{D}, \mathcal{P}^{a}(A)$ is the set of $\mu \in \mathcal{P}(A)$ which are absolutely continuous with respect to $\mathcal{L}^{D}$. In the later case, we denote by $\mathcal{P}_{2}^{a}(A)$ the intersection of $\mathcal{P}^{a}(A)$ and $\mathcal{P}_{2}(A)$.

- Assume that $\mu$ is a measure on a topological space $X$ and that $\nu$ is a measure on a topological space $Y$. We say that a Borel map t $: X \rightarrow Y$ transports $\mu$ onto $\nu$ and we write $\mathbf{t}_{\#} \mu=\nu$ if $\nu[B]=\mu\left[\mathbf{t}^{-1}(B)\right]$ for all Borel sets $B \subset Y$. We sometimes say that $\mathbf{t}$ pushes $\mu$ forward to $\nu$.

- If $h \in C^{1}\left(\mathbb{R}^{2 d}\right)$, the Hamiltonian vector field associated with $h$ is $X_{h}=J \nabla h$. When $X \in C^{1}\left([a, b] \times \mathbb{R}^{2 d}, \mathbb{R}^{2 d}\right)$ where $0 \leq a<b$, the flow of $X$ is $\Phi:[a, b] \times \mathbb{R}^{2 d} \rightarrow \mathbb{R}^{2 d}$ defined by

$$
\left\{\begin{array}{l}
\dot{\Phi}(t, z)=X(t, \Phi(t, z)) \quad t \in[a, b], \quad z \in \mathbb{R}^{2 d} \\
\Phi(0, z)=z, \quad z \in \mathbb{R}^{2 d} .
\end{array}\right.
$$

If we set $\mu_{o}=\delta_{z}$ and set $\mu_{t}=\Phi(t, \cdot)_{\#} \mu_{o}$, then

$$
\frac{d}{d t} \mu_{t}+\nabla \cdot\left(\mu_{t} X\right)=0
$$

Assume that $(X,|\cdot|)$ is a normed space, if $\mu, \nu \in \mathcal{P}_{2}(X)$, we define $\Gamma(\mu, \nu)$ to be the set of Borel probability measures on $X \times X$ which have $\mu$ and $\nu$ as their marginal, i.e. such that $\mu[A]=\gamma[A \times X], \quad \gamma[X \times B]=\nu[B]$ for all Borel sets $A, B \subset X$. We call any element of $\Gamma(\mu, \nu)$ a transport scheme for $\mu$ and $\nu$. The Wasserstein distance $W_{2}(\mu, \nu)$ between $\mu$ and $\nu$ is defined by

$$
W_{2}^{2}(\mu, \nu):=\inf _{\gamma}\left\{\int_{X \times X}|x-y|^{2} d \gamma(x, y): \gamma \in \Gamma(\mu, \nu)\right\} .
$$

Properties of the metric $W_{2}$ can be found in [2].

Any minimizer $\gamma_{o}$ in (6) is called an optimal transfer plan between $\mu$ and $\nu$. We write $\gamma_{o} \in \Gamma_{o}(\mu, \nu)$. 
Assume that $\mu$ is a Borel probability measure on $X=\mathbb{R}^{D}$ that vanishes on $(D-1)$-rectifiable sets. Then for any $\nu \in \mathcal{P}_{2}\left(\mathbb{R}^{D}\right), \Gamma_{o}(\mu, \nu)$ is a singleton, i.e. there exists a unique minimizer $\gamma_{o}$ in (6). That minimizer is characterized by the fact that $\gamma_{o}=\left(\mathbf{i d} \times \mathbf{t}_{\mu}^{\nu}\right)_{\#} \mu$ for some map $\mathbf{t}_{\mu}^{\nu}: \mathbb{R}^{D} \rightarrow \mathbb{R}^{D}$ which is the gradient of a convex function. The map $\mathbf{t}_{\mu}^{\nu}$ is the optimal map that pushes $\mu$ onto $\nu$.

We next demonstrate the various interpretations of the Hamiltonian system (1). We endow $\mathcal{P}_{2}\left(\mathbb{R}^{2 d}\right)$, the set of Borel probability measures on $\mathbb{R}^{2 d}$ with second moment bounded, with the Wasserstein distance $W_{2}$, defined in (6). A sequence $\left\{\mu^{n}\right\}_{n=1}^{\infty} \subset \mathcal{P}_{2}\left(\mathbb{R}^{2 d}\right)$ converges in the Wasserstein distance to $\mu$ for the $W_{2}$ metric if and only if $\left\{\mu^{n}\right\}_{n=1}^{\infty}$ converges narrowly to $\mu$ and we have convergence of the second moment:

$$
\int_{\mathbb{R}^{2 d}}|z|^{2} d \mu^{n}(z) \rightarrow \int_{\mathbb{R}^{2 d}}|z|^{2} d \mu(z)
$$

We refer the reader to [2] remark 7.1.11 which is one of the many sources where one can find the proof of that statement.

When $\left\{\nu^{n}\right\}_{n=1}^{\infty}=\left\{1 / n \sum_{i=1}^{n} \delta_{c_{i}^{n}}\right\}_{n=1}^{\infty}$ converges to a probability measure $\nu$ in the $W_{2}$ metric and $\left\{\mu^{n}\right\}_{n=1}^{\infty}=\left\{1 / n \sum_{i=1}^{n} \delta_{z_{i}}\right\}_{n=1}^{\infty}$ converges to a probability measure $\mu$ in the $W_{2}$ metric then, as $n$ tends to $+\infty$,

$$
H^{n}\left(z_{1}, \cdots, z_{n}\right)=1 / 2 W_{2}^{2}\left(\mu^{n}, \nu^{n}\right) \rightarrow H(\mu)=1 / 2 W_{2}^{2}(\mu, \nu) .
$$

When $d=1$ and $\nu=\chi_{Q} \mathcal{L}^{2}$, where $Q \subset \mathbb{R}^{2}$ is an open set and $\mathcal{L}^{2}$ is the Lebesgue measure on $\mathbb{R}^{2}$, then $H$ is the Hamiltonian for the semigeostrophic system. If instead,

$$
c_{i}^{n}=\left(\frac{i}{n}-\frac{1}{2}-\frac{1}{2 n}, 0\right) \quad i=1, \cdots, n
$$

(are one-dimensional), setting $z_{i}=\left(x_{i}, v_{i}\right)$, we observe that

$$
\begin{aligned}
\left|\mathbf{z}-\mathbf{c}^{n, \sigma}\right|^{2} & =\sum_{i=1}^{n} v_{i}^{2}+\sum_{i=1}^{n}\left(x_{i}-\frac{\sigma(i)}{n}+\frac{n+1}{2 n}\right)^{2} \\
& =\sum_{i=1}^{n}\left(v_{i}^{2}+x_{i}^{2}\right)+\sum_{i=1}^{n}\left(\frac{\sigma(i)}{n}-\frac{n+1}{2 n}\right)^{2}-2<\mathbf{x} ; \frac{\sigma}{n}-\frac{n+1}{2 n}>.
\end{aligned}
$$

Here, we have set $\sigma=(\sigma(1), \cdots, \sigma(n))$. The von Neumann inequality gives us that the minimum in (1) is attained for $\sigma_{\mathbf{z}}$ such that $\mathbf{x}^{\sigma_{\mathbf{z}}^{-1}}$ is the nondecreasing rearrangement of $\mathbf{x}$. Thus,

$$
\frac{1}{4 n^{2}} \sum_{i, j=1}^{n}\left|x_{i}-x_{j}\right|=\frac{1}{n}<\mathbf{x} ; \frac{\sigma_{\mathbf{z}}}{n}-\frac{n+1}{2 n}>.
$$


Also,

$$
\begin{aligned}
\frac{1}{2 n} \sum_{i=1}^{n}\left(\frac{\sigma_{\mathbf{z}}(i)}{n}-\frac{n+1}{2 n}\right)^{2} & =\frac{1}{2 n}\left(\frac{1}{n^{2}} \sum_{i=1}^{n} i^{2}+\frac{(n+1)^{2}}{4 n}-\frac{n+1}{n^{2}} \sum_{i=1}^{n} i\right) \\
& =\frac{1}{2 n} \frac{1}{12}\left(n+3-\frac{1}{n}\right) .
\end{aligned}
$$

We combine (7), (8) and (9) to conclude that

$$
H^{n}\left(z_{1}, \cdots, z_{n}\right)=\frac{|\mathbf{z}|^{2}}{2 n}-\frac{1}{4 n^{2}} \sum_{i, j=1}^{n} G\left(x_{i}-x_{j}\right)+\frac{1}{24}\left(1+3 / n-1 / n^{2}\right),
$$

where $G(x)=|x|$ is the 1-dimensional Green's function for the heat equation. The computations which led to the expression of $H^{n}$ are slight modifications of computations made by Brenier in [6], an unpublished paper. Since $\left\{1 / n \sum_{i=1}^{n} \delta_{c_{i}^{n}}\right\}_{n=1}^{\infty}$ converges in the $W_{2}$ sense to the one-dimensional Lebesgue measure restricted to the interval $\left(-\frac{1}{2}, \frac{1}{2}\right)$, then as $n$ tends to $+\infty$, $H^{n}\left(z_{1}, \cdots, z_{n}\right)$ converges to

$$
H(\mu)=\int_{\mathbb{R}^{2}} \frac{|z|^{2}}{2} d \mu(x, v)-\frac{1}{4} \int_{\mathbb{R}} G * \mu_{1} d \mu_{1}(x)-\frac{1}{24},
$$

where $\mu_{1}$ is the first marginal of $\mu$. In other words,

$$
\int_{\mathbb{R}^{2}} \frac{|z|^{2}}{2} d \mu(x, v)-\frac{1}{4} \int_{\mathbb{R}} G * \mu_{1} d \mu_{1}(x)-\frac{1}{24}=W_{2}^{2}\left(\mu, \chi_{\left(-\frac{1}{2}, \frac{1}{2}\right)} \mathcal{L}^{1} \times \delta_{0}\right) .
$$

Thus, $H$ is the Hamiltonian for the 1-dimensional Vlasov-Poisson equation

$$
\begin{cases}\partial_{t} f(t, x, v)+\partial_{x}(v f(t, x, v)) & =\partial_{v}\left(f(t, x, v) \nabla_{x} \Phi(t, x)\right) \\ \partial_{x x}^{2} \Phi(t, x) & =1-\rho(t, x)\end{cases}
$$

where $\rho(t, x)=\int_{\mathbb{R}} f(t, x, v) d v$.

When $d>1$ the identity in the second line of (11) becomes nonlinear as we see next.

Vlasov-Monge-Ampère system. Conclusions similar to (10) and (11) can be reached when $d>1$. Assume that $\nu_{o}=\left(\chi_{Q} \mathcal{H}^{d}\right) \times \delta_{0}$ where $Q \subset \mathbb{R}^{d}$ is a unit cube, $0 \in \mathbb{R}^{d}$ is the origin and $\mathcal{H}^{d}$ is the $d$-dimensional Hausdorff measure. Let $\mu=f d x d v$ be an absolutely continuous probability measure on $\mathbb{R}^{2 d}$ and denote by $\mu_{1}=\rho(x) d x$ its first marginal. Let $\varphi: \mathbb{R}^{d} \rightarrow \mathbb{R}$ be a convex function such that $\left(\nabla_{x} \varphi\right)_{\#} \mu_{1}=\chi_{Q} \mathcal{H}^{d}$. Clearly, $\Phi: \mathbb{R}^{2 d} \rightarrow \mathbb{R}$ defined by $\Phi(x, v)=\varphi(x)$ is a convex function. If $F \in C_{c}\left(\mathbb{R}^{2 d}\right)$ we have

$$
\begin{aligned}
\int_{\mathbb{R}^{2 d}} F(\nabla \Phi(x, v)) d \mu(x, v) & =\int_{\mathbb{R}^{2 d}} F\left(\nabla_{x} \varphi(x), 0\right) d \mu(x, v) \\
& =\int_{\mathbb{R}^{d}} F\left(\nabla_{x} \varphi(x), 0\right) d \mu_{1}(x) \\
& =\int_{Q} F(a, 0) d a=\int_{\mathbb{R}^{2 d}} F d \nu_{o} .
\end{aligned}
$$


The equality in (12) is due to the fact that $\mu_{1}$ is the first marginal of $\mu$, and the first one in (13) is due to the fact that $\left(\nabla_{x} \varphi\right)_{\#} \mu_{1}=\chi_{Q} \mathcal{H}^{d}$. This proves that $(\nabla \Phi)_{\#} \mu=\nu_{o}$. The Monge-Kantorovich theory asserts that if $\nabla \Phi$, the gradient of a convex function, transports $\mu$ onto $\nu$, then $(\mathbf{i d} \times \nabla \Phi)_{\#} \mu$ is the unique minimizer in (6) when $X=\mathbb{R}^{2 d}$. Here, id is the identity map in $\mathbb{R}^{2 d}$. Thus, if $\mu_{2}$ is the second marginal of $\mu$, we have that

$$
\begin{aligned}
W_{2}^{2}\left(\mu, \nu_{o}\right) & =\int_{\mathbb{R}^{2 d}}|(x, v)-\nabla \Phi(x, v)|^{2} d \mu(x, v) \\
& =\int_{\mathbb{R}^{2 d}}\left(\left|x-\nabla_{x} \varphi(x)\right|^{2}+|v|^{2}\right) d \mu(x, v) \\
& =\int_{\mathbb{R}^{d}}\left|x-\nabla_{x} \varphi(x)\right|^{2} d \mu_{1}(x)+\int_{\mathbb{R}^{d}}|v|^{2} d \mu_{2}(v) .
\end{aligned}
$$

By (14)

$$
H(\mu)=1 / 2 W_{2}^{2}\left(\mu_{1}, \chi_{Q} \mathcal{H}^{d}\right)+\int_{\mathbb{R}^{d}} \frac{|v|^{2}}{2} d \mu_{2}(v) .
$$

Let $\varphi_{\rho}$ be a function depending on $\rho$, characterized by the fact that it is convex, $\nabla_{x} \varphi_{\rho}$ maps the support of $\rho$ onto $Q$ and

$$
\rho(x)=\operatorname{det} \nabla_{x x}^{2} \varphi_{\rho}(x),
$$

The infinite dimensional Hamiltonian system associated to (16) is

$$
\begin{cases}\partial_{t} f(t, x, v)+\operatorname{div}_{x}(v f(t, x, v)) & =\operatorname{div}_{v}\left(f(t, x, v) \nabla_{x} \Phi_{\rho_{t}}(t, x)\right) \\ \operatorname{det}\left[I_{d}-\nabla_{x x}^{2} \Phi_{\rho_{t}}(x)\right] & =\rho_{t}(x) .\end{cases}
$$

where $\varphi_{\rho_{t}}(x)=|x|^{2} / 2-\Phi_{\rho_{t}}(x)$ is a convex function in the $x$ variables and $I_{d}$ is the $d \times d$ identity matrix. We have obtained in (17) the analogue of the Vlasov-Monge-Ampere system,studied first by Brenier [7] and also later by Brenier and Loeper [10]. The expression $\operatorname{det} \nabla_{x x}^{2} \varphi_{\rho}(x)$ whose dependence in $\nabla_{x x}^{2} \varphi_{\rho}(x)$ is linear in the case $d=1$ becomes nonlinear for $d>1$.

In section 2 we develope the necessary tools to prove under what condition the solutions of the systems in (2) converge to solutions which are absolutely continuous with respect to Lebesgue measure. For the convenience of the reader, we summarize some of the results of section 3 at the end of this introduction. Let

$$
\mu_{t}^{n}=1 / n \sum_{i=1}^{n} \delta_{\left(x_{i}^{n}(t), v_{i}^{n}(t)\right)}
$$

where $\left(x_{i}^{n}(t), v_{i}^{n}(t)\right)$ are solutions to the differential equation (2). The main result of section 3 is that if, at time $t=0, \mu_{o}^{n}$ converges in the metric $W_{2}$ to a probability measure $\mu_{o}<<\mathcal{L}^{2 d}$ of bounded support on $\mathbb{R}^{2 d}$ as $n$ becomes large, then up to a subsequence, at each time $t \in[0, T], \mu_{t}^{n}$ converges 
in the metric $W_{2}$ to $\mu_{t}<<\mathcal{L}^{2 d}$. In fact, we show that the initial values $\left(x_{i}^{n}(0), v_{i}^{n}(0)\right)$ can be chosen randomly. To make accurate statements, we start by considering a probability measure space $(\Omega, \Sigma, \mathbb{P})$ and independent identically distributed random variables $\xi_{i}: \Omega \rightarrow \mathbb{R}^{2 d}$ such that $\xi_{i \#} \mathbb{P}=\mu_{o}$. We assume that $\mu_{o}$ is a probability measure of bounded support on $\mathbb{R}^{2 d}$ and that $T>0$. We assume that we are given sequences of finite terms $\left\{c_{i}^{n}\right\}_{i=1}^{n} \subset \mathbb{R}^{2 d}$ and there exists a constant $E>0$ such that (3) holds. We set $\nu^{n}=1 / n \sum_{i=1}^{n} \delta_{c_{i}^{n}}$. Let $\Phi^{n}:[0, T] \times \mathbb{R}^{2 n d} \rightarrow \mathbb{R}^{2 n d}$ be the flow for the Hamiltonian $n H^{n}$, where $H^{n}$ is defined in (1). We consider the empirical distributions

$$
\mu_{t}^{n, \omega}=\frac{1}{n} \sum_{i=1}^{n} \delta_{\Phi_{i}^{n}\left(t, \xi^{n}(\omega)\right)},
$$

where $\xi^{n}(\omega)=\left(\xi_{1}(\omega), \cdots, \xi_{n}(\omega)\right)$.

Theorem 1 (Summary of section 3 ). In addition to the above assumptions, we further assume that $\mu_{o}=\rho_{o} \mathcal{L}^{2 d}$ and that $\rho_{o}$ is a bounded function. We assume that $\left\{\nu^{n}\right\}_{n=1}^{\infty}$ converges to $\nu$ in the Wasserstein distance. Then there exists a $\mathbb{P}$-measurable set $\Omega^{\prime} \subset \Omega$ such that $\mathbb{P}\left[\Omega^{\prime}\right]=1$ and for every $\omega \in \Omega^{\prime}$, the following hold:

(i) $t \rightarrow \Phi^{n}\left(t, \xi^{n}(\omega)\right)$ is well defined and is absolutely continuous on $[0, T]$. (ii) (absolute continuity of the limit of the empirical measures). There exists a sequence $\left\{n_{k}(\omega)\right\}_{k=1}^{\infty}$ (depending on $\omega$ ) and for each $t \in[0, T]$, there exists a probability density $\mu_{t}^{\omega}<<\mathcal{L}^{2 d}$ such that the empirical measures $\left\{\mu_{t}^{n_{k}(\omega), \omega}\right\}_{k=1}^{\infty}$ converge to $\mu_{t}^{\omega}$ in the metric $W_{2}$.

(iii) There exists a constant $a(\omega)<+\infty$ such that $W_{2}\left(\mu_{t}^{\omega}, \mu_{s}^{\omega}\right) \leq a(\omega)|t-s|$ for all $s, t \in[0, T]$.

(iv) $\mu_{o}^{\omega}=\mu_{o}$.

(v) (semigeostrophic system in arbitrary dimension). There exists convex, uniformly Lipschitz functions $\phi_{t}^{\omega}: \mathbb{R}^{2 d} \rightarrow \mathbb{R}$ such that $\left(\nabla \phi_{t}^{\omega}\right)_{\#} \mu_{t}^{\omega}=$ $\nu$ and

$$
\frac{d}{d t} \mu_{t}^{\omega}+\operatorname{div}_{x}\left(\left(v-\nabla_{v} \phi_{t}^{\omega}\right) \mu_{t}^{\omega}\right)=\operatorname{div}_{v}\left(\left(x-\nabla_{x} \phi_{t}^{\omega}\right) \mu_{t}^{\omega}\right)
$$

in the sense of distributions.

(vi) (conservation of the Hamiltonian) We have $H\left[\mu_{t}^{\omega}\right]=H\left[\mu_{o}\right]$ for $t \in[0, T]$.

The proof of theorem 1 is provided in theorem 4 .

\section{Convergence of empirical distribution and measure preserving maps}

Throughout this section, $(\Omega, \Sigma, \mathbb{P})$ is a metric probability space. We assume that we are given $R_{o}>0$ and 
(H1) $\mu_{o}=\rho_{o} \mathcal{L}^{D} \in \mathcal{P}^{a}\left(\mathbb{R}^{D}\right)$, supported by $B_{R_{o}}$, the open ball of radius $R_{o}$, centered at the origin.

We assume that we are given a sequence of Borel maps

(H2) $\xi_{i}: \Omega \rightarrow \mathbb{R}^{D}$ that are independent and such that $\xi_{i \#} \mathbb{P}=\mu_{o}$.

It is well-known that we can approximate $\mu_{o}$ in the narrow convergence sense by the sequence of empirical distributions $\left\{\mu_{o}^{n, \omega}\right\}_{n=1}^{\infty}$, given by

$$
\mu_{o}^{n, \omega}=\frac{1}{n} \sum_{i=1}^{n} \delta_{\xi_{i}(\omega)}
$$

as shown by (19). These measures are randomly chosen. We then let the approximate initial measure $\mu_{o}^{n, \omega}$ be transported by Hamiltonian flows $\phi_{t}^{n}$ : $\mathbb{R}^{n D} \rightarrow \mathbb{R}^{n D}$ to obtain time-dependent random probability measures $\mu_{t}^{n, \omega}$. The purpose of this section is to show that since the Hamiltonian flows are measure-preserving, under appropriate assumptions, if $\mu_{o}<<\mathcal{L}^{D}$ with an $L^{\infty}$ density, then up to a subsequence (which depends on $\omega$ ), $\left\{\mu_{t}^{n, \omega}\right\}_{n=1}^{\infty}$ converges narrowly to a measure $\mu_{t}^{\omega}$ which is absolutely continuous with respect to $\mathcal{L}^{D}$. It is not a loss of great generality to first consider $\phi_{t}^{n}$ : $\mathbb{R}^{n D} \rightarrow \mathbb{R}^{n D}$ which are time independent. Later, we also comment on necessary conditions that need to be imposed, for our conclusions to hold.

We consider a family of maps $\phi^{n}: \mathbb{R}^{n D} \rightarrow \mathbb{R}^{n D}$ which preserve Lebesgue measure in the sense that

$$
\phi_{\#}^{n} \mathcal{L}^{n D}=\mathcal{L}^{n D},
$$

for each $n$ positive integer. We define a new sequence of empirical distributions

$$
\mu^{n, \omega}=\frac{1}{n} \sum_{i=1}^{n} \delta_{\phi_{i}^{n}\left(\xi^{n}(\omega)\right)}
$$

where $\phi_{i}^{n}$ are the components of $\phi^{n}$, i.e. $\phi^{n}=\left(\phi_{1}^{n}, \cdots, \phi_{n}^{n}\right)$ and

$$
\xi^{n}=\left(\xi_{1}, \cdots, \xi_{n}\right) .
$$

Note that $\left\{\phi_{1}^{n}\left(\xi^{n}\right), \cdots, \phi_{n}^{n}\left(\xi^{n}\right)\right\}$ may not be independent and this is a source of complication while studying the points of accumulations of the sequences $\left\{\mu^{n, \omega}\right\}_{n=1}^{\infty}$. In this section, we partially characterize these points of accumulation for the narrow topology.

In the simple case where the $\phi^{n}$ 's are the identity map on $\mathbb{R}^{n D}$ and $\varphi \in$ $C\left(\mathbb{R}^{D}\right)$ is a bounded function, then, by the Strong Law of Large Numbers,

$$
\int_{\mathbb{R}^{D}} \varphi d \mu^{n, \omega}=\frac{1}{n} \sum_{i=1}^{n} \varphi\left(\xi_{i}(\omega)\right) \rightarrow \mathbb{E}\left(\varphi \circ \xi_{1}\right)=\int_{\mathbb{R}^{D}} \varphi d \mu_{o}
$$

for $\mathbb{P}$-almost every $\omega$. Here, $\mathbb{E}\left(\varphi \circ \xi_{1}\right)$ is the expectation of $\varphi \circ \xi_{1}$ and we have used that $\left\{\varphi \circ \xi_{i}\right\}_{i=1}^{\infty}$ is a collection of independent identically distributed random variables. However, the set where (19) fails, is a set of $\mathbb{P}$-zero 
measures, which depends on $\varphi$. We use (H1) and (H2), together with the fact that $C\left(\bar{B}_{R_{o}}\right)$ is separable to find a set $\Omega^{\prime} \subset \Omega$ such that $\mathbb{P}\left[\Omega^{\prime}\right]=1$ and (19) holds for all $\omega \in \Omega^{\prime}, \varphi \in C\left(\mathbb{R}^{D}\right)$. In other words, for each $\omega \in \Omega^{\prime}$,

$$
\left\{\mu^{n, \omega}\right\}_{n=1}^{\infty} \text { converges narrowly to } \mu_{o}
$$

as $n$ tends to $+\infty$.

We next note that we cannot expect that $\mu_{1}<<\mathcal{L}^{D}$ unless more restrictions can be placed on $\phi^{n}$.

1. The $L^{\infty}{ }_{-n o r m}$ may be increased unless there are extra assumptions on $\phi$.

$\overline{\text { If } T, S} \in C^{1}\left(\mathbb{R}^{D}, \mathbb{R}^{D}\right)$ are two Borel maps, inverse of each other and we set

$$
\phi_{1}^{n}=T, \phi_{2}^{n}=S, \phi_{3}^{n}=T, \phi_{4}^{n}=S, \cdots,
$$

for $n$ even. The previous reasoning can be adapted to prove that

$$
\left\{\mu^{n, \omega}\right\}_{n=1}^{\infty} \quad \text { converges narrowly to }\left(T_{\#} \mu_{o}+S_{\#} \mu_{o}\right) / 2=\mu_{1}
$$

as $n$ tends to $+\infty$. By (21), $\mu_{1}=\rho_{1} \mathcal{L}^{D}$ and we may have chosen $T$ such that

$$
\left\|\rho_{1}\right\|_{L^{\infty}} \geq \frac{\left\|\rho_{o}\right\|_{L^{\infty}\left(B_{R_{o}}\right)}}{2\|\operatorname{det} \nabla T\|_{L^{\infty}\left(B_{R_{o}}\right)}}
$$

and so, in general, one cannot expect that $\left\|\rho_{1}\right\|_{L^{\infty}\left(T\left(B_{R_{o}}\right) \cup S\left(B_{R_{o}}\right)\right)} \leq$ $\left\|\rho_{o}\right\|_{L^{\infty}\left(B_{R_{o}}\right)}$.

2. Concentration to a Dirac mass may occur unless there are extra assumptions on $\phi$.

When

$$
\begin{aligned}
\phi_{1}^{n}\left(z_{1}, \cdots, z_{n}\right) & =2^{n(n-1)} z_{1}, \phi_{2}^{n}\left(z_{1}, \cdots, z_{n}\right) \\
& =\frac{z_{2}}{2^{n}}, \cdots, \phi_{n}^{n}\left(z_{1}, \cdots, z_{n}\right)=\frac{z_{n}}{2^{n}},
\end{aligned}
$$

one can readily check that

$$
\left\{\mu^{n, \omega}\right\}_{n=1}^{\infty} \text { converges narrowly to } \delta_{0} .
$$

(23) shows that one cannot expect that $\mu_{1}<<\mathcal{L}^{D}$ unless more restrictions, such as we give in (24) below, are imposed on $\phi^{n}$. Under this additional assumption, we learnt from (21) that even if we succeed in proving that $\mu_{1}=\rho_{1} \mathcal{L}^{D}<<\mathcal{L}^{D}$ we cannot expect a control of the $L^{\infty}$-norm of the form $\left\|\rho_{o}\right\|_{L^{\infty}} \geq\left\|\rho_{1}\right\|_{L^{\infty}}$. In fact, (22) suggest that $\left\|\rho_{1}\right\|_{L^{\infty}}$ may become very large.

Suppose that there exists $R_{1} \geq 1 / 2$ such that

$$
\left|\phi_{i}^{n}\left(z_{1}, \cdots, z_{n}\right)\right| \leq R_{1} \quad(i=1, \cdots, n, \quad \text { and } \quad n=1, \cdots),
$$


for all $z_{1} \in B_{R_{o}}, \cdots, z_{n} \in B_{R_{o}}$. In section 3 , we show that this can be proved for the semigeostrophic shallow water system. The aim in this section is to prove under this condition that if up to a subsequence which depends on $\omega,\left\{\mu^{n, \omega}\right\}_{n=1}^{\infty}$ converges narrowly to $\mu^{\omega} \mathbb{P}$-almost everywhere, then $\mu^{\omega}<<$ $\mathcal{L}^{D}$. To achive that goal, we state two intermediary lemmas.

Lemma 1. Assume that $\mu_{o}=\rho_{o} \mathcal{L}^{D}$ and $\left\{\xi_{i}\right\}_{i=1}^{\infty}$ are such that $\rho_{o} \in L^{\infty}$ and that (H1), (H2) hold. Let $C_{o}=\max \left\{|| \rho_{o} \|_{L^{\infty}}, 1 / 2\left|Q_{R_{1}}\right|\right\}$ and $\phi^{n}: \mathbb{R}^{n D} \rightarrow$ $\mathbb{R}^{n D}$ be Borel maps which preserve Lebesgue measure in the sense that (18) holds and assume that (24) holds (with $2 R_{1} \geq 1$ ). If up to a subsequence (which depends on $\omega),\left\{\mu^{n, \omega}\right\}_{n=1}^{\infty}$ converges narrowly to $\mu^{\omega} \mathbb{P}$-almost everywhere, then for all Borel sets $A \subset \mathbb{R}^{D}, \mathbb{P}\left\{\omega \in \Omega: \mu^{\omega}[A]>p\right\}=0$ whenever $p \in(0,1)$ and $2 C_{o}\left|Q_{R_{1}} \| A\right|^{p}<1$.

Proof. 1. We first claim that for each Borel set $A \subset \mathbb{R}^{D}$ such that $|A| \leq 1$, for any real number $p \in(0,1)$ and integer $n \geq 1$,

$$
\mathbb{P}\left\{\omega \in \Omega: \mu^{n, \omega}[A] \geq p\right\} \leq\left(2 C_{o}\left|Q_{R_{1}}\right|^{1-p}|A|^{p}\right)^{n}
$$

where $Q_{R_{1}}=\left[-R_{1}, R_{1}\right] \times \cdots \times\left[-R_{1}, R_{1}\right] \subset \mathbb{R}^{D}$. We prove this claim only for $p$ such that $p n$ is not an integer. The proof in the case where $p n$ is an integer follows the same line of argument. Let $q=[p n]+1$ where $[\cdot]$ is the greatest integer part function. Set

$$
I=\left\{i=\left(i_{1}, \cdots, i_{q}\right) \in \mathbf{N}: 1 \leq i_{1}<i_{2}<\cdots, i_{q} \leq n\right\}
$$

For $i \in I$ we denote by $Q^{i}$ the subset of $\mathbb{R}^{n D}$ of the form $A_{1} \times A_{2} \times \cdots A_{n}$ where,

$$
A_{j}= \begin{cases}A & j=i_{1}, \cdots, i_{q} \\ Q_{R_{1}} & j \neq i_{1}, \cdots, i_{q} .\end{cases}
$$

Since $\mu^{n, \omega}[A] \in\left\{0, \frac{1}{n}, \cdots, \frac{n-1}{n}, 1\right\}$ and $p n$ is not an integer then $\mu^{n, \omega}[A] \geq p$ is equivalent to $\mu^{n, \omega}[A] \geq q / n$. Hence,

$$
\begin{aligned}
\mathbb{P}\left\{\omega \in \Omega: \mu^{n, \omega}[A] \geq p\right\} & =\mathbb{P}\left\{\omega \in \Omega: \mu^{n, \omega}[A] \geq q / n\right\} \\
& =\mathbb{P}\left\{\omega \in \Omega: \exists i \in I, \phi^{n}\left(\xi^{n}(\omega)\right) \in Q^{i}\right\} \\
& \leq \sum_{i \in I} \mathbb{P}\left\{\omega \in \Omega: \phi^{n}\left(\xi^{n}(\omega)\right) \in Q^{i}\right\} \\
& \leq \sum_{i \in I} \mathbb{P}\left\{\omega \in \Omega: \xi^{n}(\omega) \in\left(\phi^{n}\right)^{-1}\left[Q^{i}\right]\right\}
\end{aligned}
$$


This, together with the facts that (18) holds and the $\xi_{i}$ 's are independent yields that

$$
\begin{aligned}
\mathbb{P}\left\{\omega \in \Omega: \mu^{n, \omega}[A] \geq p\right\} & \leq \sum_{i \in I} \mu_{o} \times \cdots \times \mu_{o}\left[\left(\phi^{n}\right)^{-1}\left[Q^{i}\right]\right] \\
& \leq \sum_{i \in I}\left\|\rho_{o}\right\|_{L^{\infty}}^{n} \mathcal{L}^{n D}\left[\left(\phi^{n}\right)^{-1}\left[Q^{i}\right]\right] \\
& =\sum_{i \in I}\left\|\rho_{o}\right\|_{L^{\infty}}^{n} \mathcal{L}^{n D}\left[Q^{i}\right] \\
& \leq 2^{n}\left\|\rho_{o}\right\|_{L^{\infty}}^{n}\left|Q_{R_{1}}\right|^{n-q}|A|^{q} \\
& \leq 2^{n}\left\|\rho_{o}\right\|_{L^{\infty}}^{n}\left|Q_{R_{1}}\right|^{n-n p}|A|^{n p}
\end{aligned}
$$

To obtain the last inequality in (26) we have used that $|A| \leq 1$ and $\left|Q_{R_{1}}\right| \geq$ 1. This proves (25) with $C_{o}=\left\|\rho_{o}\right\|_{L^{\infty}}$.

2. Let $\Omega_{1}$ be a subset of $\Omega$ such that $\mathbb{P}\left[\Omega_{1}\right]=1$ and for all $\omega \in \Omega_{1}$, there exists an increasing, unbounded sequence $\left\{n_{k}(\omega)\right\}_{k=1}^{\infty}$ such that

$$
\left\{\mu^{n_{k}(\omega), \omega}\right\}_{k=1}^{\infty} \quad \text { converges narrowly to } \mu^{\omega}
$$

as $k$ tends to $+\infty$. Let $A$ be a Borel subset of $\mathbb{R}^{D}$ such that $2 C_{o}\left|Q_{R_{1}}\right||A|^{p}<$ 1. Since $\left|Q_{R_{1}}\right| \geq 1$ we conclude that

$$
2 C_{o}\left|Q_{R_{1}}\right|^{1-p}|A|^{p}<1 .
$$

For $\epsilon>0$, thanks to (28), we may choose an open set $A_{\epsilon}$ containing $A$ and such that

$$
\left|A_{\epsilon}\right|<|A|+\epsilon<1, \quad 2 C_{o}\left|Q_{R_{1}}\right|^{1-p}\left|A_{\epsilon}\right|^{p}<1 .
$$

We use (27) to conclude that

$$
\liminf _{k \rightarrow+\infty} \mu^{n_{k}(\omega), \omega}\left[A_{\epsilon}\right] \geq \mu^{\omega}\left[A_{\epsilon}\right] .
$$

Consequently, for each integer $k>1$,

$$
\left\{\omega \in \Omega_{1}: \mu^{\omega}\left[A_{\epsilon}\right]>p\right\} \subset \cup_{n=k}^{\infty}\left\{\omega \in \Omega_{1}: \mu^{n, \omega}\left[A_{\epsilon}\right] \geq p\right\} .
$$

Since by (29) $\left|A_{\epsilon}\right|<1$, we conclude that (25) holds for $A_{\epsilon}$ in place of $A$. We could conclude the proof of the lemma here by simply invoking the BorelCantelli lemma. However, for those not familiar with the Borel-Cantelli lemma, let us detail the arguments yielding the proof. We use (25) with $A_{\epsilon}$ in place of $A$, together with (30), to conclude that

$$
\begin{aligned}
\mathbb{P}\left\{\omega \in \Omega: \mu^{\omega}\left[A_{\epsilon}\right]>p\right\} & \leq \sum_{n=k}^{\infty}\left(2 C_{o}\left|Q_{R_{1}}\right|^{1-p}\left|A_{\epsilon}\right|^{p}\right)^{n} \\
& =\frac{\left(2 C_{o}\left|Q_{R_{1}}\right|^{1-p}\left|A_{\epsilon}\right|^{p}\right)^{k}}{1-\left(2 C_{o}\left|Q_{R_{1}}\right|^{1-p}\left|A_{\epsilon}\right|^{p}\right)} .
\end{aligned}
$$


Hence,

$$
\mathbb{P}\left\{\omega \in \Omega: \mu^{\omega}[A]>p\right\} \leq \frac{\left(2 C_{o}\left|Q_{R_{1}}\right|^{1-p}\left|A_{\epsilon}\right|^{p}\right)^{k}}{1-\left(2 C_{o}\left|Q_{R_{1}}\right|^{1-p}\left|A_{\epsilon}\right|^{p}\right)} .
$$

We let first $\epsilon$ tend to 0 in (31), then $k$ tends to $+\infty$ in the subsequent inequality to conclude the proof of the lemma.

Warning. The inequality in (25) yields that

$$
\mathbb{P}\left\{\omega \in \Omega: \mu^{n, \omega}[A] \geq p\right\}=0
$$

whenever $A \subset \mathbb{R}^{D}$ and $|A|=0$. This is not sufficient to give the conclusion that $\mu^{n, \omega}<<\mathcal{L}^{D}$. Indeed, let us consider the family of measures

$$
\nu^{n, \omega}=\delta_{\xi_{1}(\omega)},
$$

which does not satisfy the assumptions in lemma 1, but are used just to illustrate that (32) cannot be used as a short cut to the conclusions of the theorem. Clearly, if $p \in(0,1)$, since $\nu^{n, \omega}[A] \geq p$ is equivalent to $\nu^{n, \omega}[A]=1$ we conclude that

$$
\begin{aligned}
\mathbb{P}\left\{\omega \in \Omega: \nu^{n, \omega}[A] \geq p\right\} & =\mathbb{P}\left\{\omega \in \Omega: \nu^{n, \omega}[A]=1\right\} \\
& =\mathbb{P}\left\{\omega \in \Omega: \xi_{1}(\omega) \in A\right\}=\mu_{o}[A] .
\end{aligned}
$$

Thus (32) holds although $\nu^{n, \omega}<<\mathcal{L}^{D}$ fails.

Lemma 2. Assume that $\left\{\mu^{\omega}\right\}_{\omega \in \Omega}$ is a family of Borel probability measures on $\mathbb{R}^{D}$. Assume that there exists a constant $C>1$ such that for all Borel sets $A \subset \mathbb{R}^{D}, \mathbb{P}\left\{\omega \in \Omega: \mu^{\omega}[A]>p\right\}=0$ whenever $p \in(0,1)$ and $2 C|A|^{p}<1$. Then, there exists a $\mathbb{P}$-measurable set $\Omega^{\prime} \subset \Omega$ such that $\mathbb{P}\left[\Omega^{\prime}\right]=1$ and

$$
\mu^{\omega}[A] \leq \frac{\ln (2 C)}{-\ln |A|}
$$

for all $\omega \in \Omega^{\prime}$, and all $A \subset \mathbb{R}^{D}$ such that $2 C|A|<1$. Here, we have denoted by $|A|$ the Lebesgue measure $\mathcal{L}^{D}(A)$ of $A$.

Proof. 1. We first prove a weaker statement. Let $A \subset \mathbb{R}^{D}$ be a Borel set such that $0<2 C|A|<1$. Set

$$
p=\frac{\ln (2 C)}{-\ln |A|} .
$$

We use that $C>1$ to conclude that $p \in(0,1)$. Since $2 C|A|^{p}=1$ we may find a sequence $\left\{p_{n}\right\}_{n=1}^{\infty} \subset(0,1)$ decreasing to $p$ and such that $2 C|A|^{p_{n}}<1$. For each $n$, there exists a set $N_{n} \subset \Omega$ such that $\mathbb{P}\left[N_{n}\right]=0$ and $\mu^{\omega}[A] \leq p_{n}$ for all $\omega \in \Omega \backslash N_{n}$ and all $n \geq 1$ integer. Set

$$
N(A)=\cup_{n=1}^{\infty} N_{n} .
$$


We have $\mathbb{P}[N(A)]=0$ and $\mu^{\omega}[A] \leq p_{n}$ for all $\omega \in \Omega \backslash N(A)$. Letting $n$ tends to $+\infty$, we conclude that

$$
\mu^{\omega}[A] \leq p=\frac{\ln (2 C)}{-\ln |A|}
$$

for all $\omega \in \Omega \backslash N(A)$.

2. We next select a specific countable basis for the standard topology of $\mathbb{R}^{D}$. Let $\mathcal{R}_{1}$ be the set of rectangles in $\mathbb{R}^{D}$, whose vertices are rational numbers. Any rectangle being completely determined by $D+1$ of its vertices, we clearly have that $\mathcal{R}_{1}$ is countable since it can be imbedded in $\mathbb{Q}^{D(D+1)}$. We conclude that the set $\mathcal{R}_{k}$ which consists of subsets of $\mathbb{R}^{D}$ which are $k$-unions of subsets of $\mathcal{R}_{1}$, is countable. Thus,

$$
\mathcal{R}=\cup_{k=1}^{\infty} \mathcal{R}_{k}
$$

is also countable. We denote by $\mathcal{A}$ the set of $A \in \mathcal{R}$ such that $2 C|A|<1$. For each of these $A$, we define $\Omega(A)=\Omega \backslash N(A)$ and we set

$$
\Omega^{\prime}=\cap_{A \in \mathcal{A}} \Omega(A) \text {. }
$$

Since $\mathcal{A}$ is countable and by assumption $\mathbb{P}[\Omega(A)]=1$ for each $A \in \mathcal{A}$, we conclude that $\mathbb{P}\left[\Omega^{\prime}\right]=1$.

3. Let $K \subset \mathbb{R}^{D}$ be a compact set such that $2 C|K|<1$. There exists a sequence $R_{n} \in \mathcal{A}$ such that $K \subset R_{n}$ and $\left|R_{n}\right|<|K|+1 / n$. Thus, for $\omega \in \Omega^{\prime}$ and $n$ large enough so that $2 C(|K|+1 / n)<1$, we have

$$
\mu^{\omega}[K] \leq \mu^{\omega}\left[R_{n}\right] \leq \frac{\ln (2 C)}{-\ln \left|R_{n}\right|} \leq \frac{\ln (2 C)}{-\ln (|K|+1 / n)} .
$$

Letting $n$ tend to $+\infty$ in the previous inequalities, we conclude that

$$
\mu^{\omega}[K] \leq \frac{\ln (2 C)}{-\ln |K|} .
$$

4. Let $\omega \in \Omega^{\prime}$ and let $A \subset \mathbb{R}^{D}$ be a Borel set such that $2 C|A|<1$. Since $\mu^{\omega}$ is a Radon measure, for each integer $n$ there exists a compact set $K_{n} \subset \mathbb{R}^{D}$ such that $K_{n} \subset A$ and $\mu^{\omega}[A]<\mu^{\omega}\left[K_{n}\right]+1 / n$. Hence, $2 C\left|K_{n}\right|<1$ and so, using (34), we have

$$
\mu^{\omega}[A]<\mu^{\omega}\left[K_{n}\right]+1 / n \leq \frac{\ln (2 C)}{-\ln \left|K_{n}\right|}+1 / n \leq \frac{\ln (2 C)}{-\ln |A|}+1 / n .
$$

Letting $n$ tend to $+\infty$, we conclude the proof of the lemma.

Remark 1. Assume that $\mu$ is a probability measure on $\mathbb{R}^{D}$ such that $\mu[A] \leq C /|\ln | A||$ for, say, all $|A| \leq 1 / 2$. Then $\mu=\rho \mathcal{L}^{D}$ for some $\rho$ : $\mathbb{R}^{d} \rightarrow[0,+\infty]$ such that

$$
\int_{\mathbb{R}^{D}} \varphi(\rho) \rho d x \leq \varphi(2)-\varphi(0)+C \int_{2}^{\infty} \frac{\varphi^{\prime}(s)}{\ln s} d s .
$$


Here, $\varphi:[0,+\infty) \rightarrow[0,+\infty)$ is any increasing function. For instance one can choose $\varphi(t)=(\ln t)^{1-\epsilon}$ for $t$ large, where $\epsilon \in(0,1)$. Indeed,

$$
\int_{\mathbb{R}^{D}} \varphi(\rho) \rho d x=\int_{\mathbb{R}^{D}} \varphi(\rho) d \mu=\int_{0}^{\infty} \mu\{\varphi(\rho)>t\} d t=\int_{0}^{\infty} \mu\{\rho>s\} \varphi^{\prime}(s) d s .
$$

For $s \geq 2$, if we set $A=A_{s}:=\{\rho>s\}$, Markov's inequality gives $2\left|A_{s}\right| \leq$ $s\left|A_{s}\right|<1$. This, together with (35), yields

$\int_{\mathbb{R}^{D}} \varphi(\rho) \rho d x \leq \int_{0}^{2} \varphi^{\prime}(s) d s+\int_{2}^{\infty} \frac{C \varphi^{\prime}(s)}{|\ln | A_{s}||} d s \leq \varphi(2)-\varphi(0)+C \int_{2}^{\infty} \frac{\varphi^{\prime}(s)}{\ln s} d s$.

This completes the proof of the remark.

The main theorem of this section is the following.

Theorem 2 (Absolute continuity of the limit of the empirical distributions). Assume that $\mu_{o}=\rho_{o} \mathcal{L}^{D}$ is such that $\rho_{o} \leq C_{o}$ for a constant $C_{o}>1 /\left|Q_{R_{1}}\right|$. Assume that (H1), (H2) hold and that $2 R_{1} \geq 1$. Let $\phi^{n}: \mathbb{R}^{n D} \rightarrow \mathbb{R}^{n D}$ be Borel maps which preserve Lebesgue measure in the sense that (18) holds and assume that (24) holds. Assume there exists a subsequence $\left\{n_{k}(\omega)\right\}_{k=1}^{\infty}$ (which depends on $\omega$,) such that $\left\{\mu^{n_{k}(\omega), \omega}\right\}_{k=1}^{\infty}$ converges narrowly to $\mu^{\omega} \mathbb{P}$-almost everywhere. Then there exists a $\mathbb{P}$ measurable set $\Omega^{\prime} \subset \Omega$ such that $\mathbb{P}\left[\Omega^{\prime}\right]=1$ and

$$
\mu^{\omega}[A] \leq \frac{\ln \left(4 C_{o}\left|Q_{R_{1}}\right|\right)}{-\ln |A|}
$$

for all Borel sets $A \subset \mathbb{R}^{D}$ such that $4 C_{o}\left|Q_{R_{1}}\right||A|<1$.

Proof. The proof of this theorem is a direct consequence of lemmas 1 and 2.

Remark 2. Let $\Omega^{\prime}$ be the set found in theorem 2 . By remark $1, \mu^{\omega}<<\mathcal{L}^{D}$ for each $\omega \in \Omega^{\prime}$.

\section{Convergence of the spatially discrete scheme}

Throughout this section, $(\Omega, \Sigma, \mathbb{P})$ is a metric probability space. We assume that

$$
\text { (C1) } \mu_{o}=\rho_{o} \mathcal{L}^{2 d} \in \mathcal{P}_{2}^{a}\left(\mathbb{R}^{2 d}\right)
$$

We assume that we are given a sequence of Borel maps

(C2) $\xi_{i}: \Omega \rightarrow \mathbb{R}^{2 d}$ that are are independent and such that $\xi_{i \#} \mathbb{P}=\mu_{o}$.

The variant of the de la Vallée-Poussin lemma proven in [11] (page 835836), together with (C1) gives a nonnegative, convex, increasing function 
$\zeta \in C^{1}([0,+\infty))$ with the property that $\zeta(0)=0, \lim _{t \rightarrow+\infty} \zeta(t) / t=+\infty$, $\left\|\zeta^{\prime \prime}\right\|_{L^{\infty}} \leq 1$ and

$$
\int_{\mathbb{R}^{2 d}} \zeta\left(|z|^{2}\right) d \mu_{o}(z)<+\infty .
$$

It is easy to see that we can approximate $\mu_{o}$ in the narrow convergence sense by a sequence of discrete probability measures $\left\{\mu_{o}^{n, \omega}\right\}_{n=1}^{\infty}$ chosen randomly and given by

$$
\mu_{o}^{n, \omega}=\frac{1}{n} \sum_{i=1}^{n} \delta_{\left(\xi_{i}(\omega)\right)} .
$$

More precisely, the Strong Law of Large Numbers ensures that for $\mathbb{P}$-almost every $\omega \in \Omega,\left\{\mu_{o}^{n, \omega}\right\}_{n=1}^{\infty}$ converges narrowly to $\mu_{o}$ and the second moments of $\left\{\mu_{o}^{n, \omega}\right\}_{n=1}^{\infty}$ converge to the second moments of $\mu_{o}$. In other words, for $\mathbb{P}$-almost every $\omega \in \Omega$,

$$
\lim _{n \rightarrow+\infty} W_{2}\left(\mu_{o}^{n, \omega}, \mu_{o}\right)=0 .
$$

As in the introduction, we first fix $n$ distinct points $c_{1}^{n}=\left(a_{1}^{n}, b_{1}^{n}\right), \cdots$, $c_{n}^{n}=\left(a_{n}^{n}, b_{n}^{n}\right) \in \mathbb{R}^{2 d}$ and consider the Hamiltonians $H^{n}: \mathbb{R}^{2 n d} \rightarrow \mathbb{R}$ defined by

$$
H^{n}\left(z_{1}, \cdots, z_{n}\right)=\frac{1}{2 n} \min _{\sigma \in S_{n}}\left\|\mathbf{z}-\mathbf{c}^{n, \sigma}\right\|^{2}
$$

where $S_{n}$ is the set of permutations of $n$ letters, we have used the notation

$$
\mathbf{c}^{n, \sigma}=\left(c_{\sigma(1)}^{n}, \cdots, c_{\sigma(n)}^{n}\right), \quad \mathbf{z}=\left(z_{1}, \cdots, z_{n}\right),
$$

and the Euclidean norm of $\mathbf{z}-\mathbf{c}^{n, \sigma}$ is denoted by $\left\|\mathbf{z}-\mathbf{c}^{n, \sigma}\right\|$. Set

$$
\nu^{n}=1 / n \sum_{i=1}^{n} \delta_{c_{i}^{n}} \quad \text { and } \quad \mu=1 / n \sum_{i=1}^{n} \delta_{z_{i}} .
$$

If we identify $\Gamma\left(\mu, \nu^{n}\right)$ with the set of bistochastic matrices, Birkoff's theorem gives that its extreme points are the permutation matrices. From this and the fact that $W_{2}^{2}\left(\mu, \nu^{n}\right)$ is the infimum of a linear functional of bistochastic matrices, it is clear that $H^{n}\left(z_{1}, \cdots, z_{n}\right)=1 / 2 W_{2}^{2}\left(\mu, \nu^{n}\right)$. Observe that

$$
H^{n}\left(z_{1}, \cdots, z_{n}\right)=\frac{\|\mathbf{z}\|^{2}+\left\|\mathbf{c}^{n}\right\|^{2}}{2 n}-\frac{1}{n} G^{n}(\mathbf{z}),
$$

where

$$
G^{n}(\mathbf{z})=\sup _{\sigma \in S_{n}}\left\langle\mathbf{z} ; \mathbf{c}^{n, \sigma}\right\rangle,
$$

is clearly a convex function as a supremum of finitely many linear functions. Hence the eigenvalues of the matrix of the second derivatives of $H^{n}$ are less than or equal to $1 / n$. Note that the extreme points of $\partial G^{n}(\mathbf{z})$ are the $\mathbf{c}^{n, \sigma}$ which satisfy $G^{n}(\mathbf{z})=\left\langle\mathbf{z} ; \mathbf{c}^{n, \sigma}\right\rangle$. When $G^{n}$ is differentiable at $\mathbf{z}, \mathbf{c}^{n, \sigma}$ is 
uniquely determined and is denoted by $\mathbf{c}^{n, \sigma_{z}}$. We define the vector field $\mathbf{b}: \mathbb{R}^{2 n d} \rightarrow \mathbb{R}^{2 n d}$ by

$$
\mathbf{b}(\mathbf{z})=-n\left(J \nabla_{z_{1}} H^{n}, \cdots, J \nabla_{z_{n}} H^{n}\right) .
$$

where we recall that $J$ is the $(2 d) \times(2 d)$ symplectic matrix defined by

$$
J=\left(\begin{array}{cc}
0 & -I_{d} \\
I_{d} & 0
\end{array}\right) .
$$

At the points of differentiability of $H^{n}$ we have

$$
\mathbf{b}(\mathbf{z})=-\left(J\left(z_{1}-c_{1}^{n, \sigma_{\mathbf{z}}}\right), \cdots, J\left(z_{n}-c_{n}^{n, \sigma_{\mathbf{z}}}\right)\right) .
$$

We have the following proposition whose first parts (i) and (ii), follow directly from section 6 of Ambrosio's theory [1]. Its last part, (iii), is then a direct consequence of (i) and (ii).

Proposition 1. Let $T>0$ be a real number. Then there exists a flow $\Phi^{n}$ : $[0,+\infty) \times \mathbb{R}^{2 n d} \rightarrow \mathbb{R}^{2 n d}$ satisfying the following condition:

(i) $\Phi^{n}(t, \cdot)_{\#} \mathcal{L}^{2 n d}=\mathcal{L}^{2 n d}$ for $t \in[0, T]$.

(ii) There exists a set $\mathcal{N}_{n} \subset \mathbb{R}^{2 n d}$ satisfying $\mathcal{L}^{2 n d}\left[\mathcal{N}_{n}\right]=0$ such that for $\mathbf{z} \in \mathbb{R}^{2 n d} \backslash \mathcal{N}_{n}, \Phi^{n}(\cdot, \mathbf{z})$ is an absolutely continuous path and $\Phi^{n}(t, \mathbf{z})=$ $\left.\mathbf{z}+\int_{0}^{t} \mathbf{b}\left(\Phi^{n}(s, \mathbf{z})\right)\right) d s$ for every $t \in[0, T]$ and $T E<R / 2$, where $E$ is the constant defined in (3).

(iii) For $\mathbf{z} \in \mathbb{R}^{2 n d} \backslash \mathcal{N}_{n}, H^{n}\left(\Phi^{n}(t, \mathbf{z})\right)=H^{n}(\mathbf{z})$.

As a consequence, we have for every $\mathbf{z} \in \mathbb{R}^{2 n d} \backslash \mathcal{N}_{n}$ and $\mathcal{L}^{1}$-almost every $t \in(0, T)$

$$
\left\{\begin{array}{l}
\dot{\Phi}^{n}(t, \mathbf{z})=\mathbf{b}\left(\Phi^{n}(t, \mathbf{z})\right) \\
\Phi^{n}(0, \mathbf{z})=\mathbf{z} .
\end{array} .\right.
$$

In other words, $\Phi^{n}$ is the flow for the Hamiltonian $n H^{n}$. The theory in [1] does not apply directly to $\mathbf{b}$ since we have $\mathbf{b} \in L_{l o c}^{\infty}\left(\mathbb{R}^{2 n d}\right)^{2 n d}$ instead of $\mathbf{b} \in L^{\infty}\left(\mathbb{R}^{2 n d}\right)^{2 n d}$. To obtain proposition 1 from the theory in [1], we use a cutoff function and approximate $H^{n}$ by

$$
H_{r}^{n}(\mathbf{z})=\sum_{i=1}^{n} \frac{l_{r}\left(\left|z_{i}\right|^{2}\right)}{2 n}+\frac{|\mathbf{c}|^{2}}{2 n}-G^{n}(\mathbf{z}),
$$

where $l_{r}(t)=r l(t / r)$ and $l \in C^{\infty}(\mathbb{R})$ is such that $|l(t)| \leq 2,0 \leq l^{\prime}(t) \leq 2$ and

$$
l(t)= \begin{cases}t & \text { for }|t| \leq 1 \\ 0 & \text { for }|t| \geq 2 .\end{cases}
$$

The theory in [1] applies to $\mathbf{b}_{r}(\mathbf{z})=-n\left(J \nabla_{z_{1}} H_{r}^{n}, \cdots, J \nabla_{z_{n}} H_{r}^{n}\right)$ to provide a flow $\Phi_{r}^{n}$, for the Hamiltonian $H_{r}^{n}$. One can readily check that the arguments which led to (45) apply to $\mathbf{b}_{r}$ and yield that

$$
\left|\Phi_{r, i}^{n}(t, \mathbf{z})\right| \leq\left|z_{i}\right|+E t .
$$


Here, we have written

$$
\begin{gathered}
\Phi^{n}(t, \mathbf{z})=\left(\Phi_{1}^{n}(t, \mathbf{z}), \cdots, \Phi_{n}^{n}(t, \mathbf{z})\right), \\
\Phi_{r}^{n}(t, \mathbf{z})=\left(\Phi_{r, 1}^{n}(t, \mathbf{z}), \cdots, \Phi_{r, n}^{n}(t, \mathbf{z})\right) .
\end{gathered}
$$

Observe that $\mathbf{b}_{r}$ coincides with $\mathbf{b}$ on $\left\{\mathbf{z} \in \mathbb{R}^{2 n D}:\left|z_{1}\right|^{2}, \cdots,\left|z_{n}\right|^{2}<r\right\}$. This, together with (41), yields that if $r$ is large enough so that $T E<$ $r / 2$, then when $\left|z_{i}\right|<r / 2$, we have that $\Phi_{r}^{n}(t, \mathbf{z})=\Phi^{n}(t, \mathbf{z})$. Letting $r$ tend to $+\infty$ we obtain the proposition. We have used (39) to obtain that $d H^{n}\left(\Phi^{n}(t, \mathbf{z})\right) / d t=0$ for $\mathcal{L}^{1}$-almost every $t \in[0, T]$ which gave (iii).

Let $\Omega_{n}$ be the set of $\omega \in \Omega$ such that $\xi^{n}(\omega) \notin \mathcal{N}_{n}$, where

$$
\xi^{n}=\xi_{1} \times \cdots \times \xi_{n} .
$$

We use $(\mathrm{C} 2)$ and the fact that $\mu_{o}<<\mathcal{L}^{2 d}$ to obtain that $\left(\xi^{n}\right)_{\#} \mathbb{P}=\mu_{o} \times$ $\cdots \times \mu_{o}<<\mathcal{L}^{2 n d}$. Since $\mathcal{L}^{2 n d}\left[\mathcal{N}_{n}\right]=0$ we conclude that $\mathbb{P}\left[\Omega_{n}\right]=1$. Set

$$
\Omega^{o}=\cap_{n=1}^{\infty} \Omega_{n} \text {. }
$$

We also define $\Omega^{\prime}$ to be the set of $\omega \in \Omega^{o}$ such that $\left\{\mu_{o}^{n, \omega}\right\}_{n=1}^{\infty}$ converges to $\mu_{o}$ in $\left(\mathcal{P}_{2}\left(\mathbb{R}^{2 d}\right), W_{2}\right)$ and

$$
\frac{1}{n} \sum_{i=1}^{n} p\left(\xi_{i}(\omega)\right) \rightarrow \int_{\mathbb{R}^{2 d}} p(z) d \mu_{o}(z)
$$

for $p: \mathbb{R}^{2 d} \rightarrow \mathbb{R}$ such that

$$
p(z) \equiv|z| \quad \text { or } \quad p(z) \equiv|z|^{2} \quad \text { or } \quad p(z) \equiv \zeta\left(|z|^{2}\right) .
$$

By (C2) and the Strong Law of Large Numbers,

$$
\mathbb{P}\left[\Omega^{\prime}\right]=1 \text {. }
$$

For each $\omega \in \Omega^{\prime}$ we set

$$
\mu_{t}^{n, \omega}=\frac{1}{n} \sum_{i=1}^{n} \delta_{\Phi_{i}^{n}\left(t, \xi^{n}(\omega)\right)} .
$$

Remark 3 ( Properties of $\Phi^{n}$ and $\mu_{t}^{n, \omega}$ ).

(i) If $\mathbf{z} \notin \mathcal{N}_{n}$ then

$$
\left|\Phi_{i}^{n}(t, \mathbf{z})\right| \leq\left|z^{i}\right|+E t,
$$

where $E$ is the constant defined in (3).

(ii) If $\omega \in \Omega^{o}$ and $0 \leq s<t \leq T$, then

$$
W_{2}\left(\mu_{t}^{n, \omega}, \mu_{s}^{n, \omega}\right) \leq(t-s) a(\omega)
$$

where $a(\omega)=\sup _{n \geq 1} 4\left(W_{2}^{2}\left(\mu_{o}^{n, \omega}, \delta_{0}\right)+(T+1)^{2} E^{2}\right)^{1 / 2}$. We use $(37)$ and the fact that by the triangle inequality

$$
W_{2}\left(\mu_{o}^{n, \omega}, \delta_{0}\right) \leq W_{2}\left(\mu_{o}^{n, \omega}, \mu_{o}\right)+W_{2}\left(\mu_{o}, \delta_{0}\right)
$$

to obtain that $a(\omega)<+\infty$. 
Proof. Using (39) we obtain that for each $i=1, \cdots, n$

$$
\partial_{t}\left|\Phi_{i}^{n}\right|=\left\langle\frac{\Phi_{i}^{n}}{\left|\Phi_{i}^{n}\right|},-J\left(\Phi_{i}^{n}-c_{i}^{\sigma_{\Phi^{n}}}\right)\right\rangle=\left\langle\frac{\Phi_{i}^{n}}{\left|\Phi_{i}^{n}\right|}, J c_{i}^{\sigma_{\Phi^{n}}}\right\rangle \leq E
$$

which proves (45). We define $V_{t}: \mathbb{R}^{2 d} \rightarrow \mathbb{R}^{2 d}$, a time dependent Borel velocity field on the support of $\mu_{t}^{n, \omega}$, by

$$
V_{t}\left(\Phi_{i}^{n}(t, \omega)\right)=\mathbf{b}_{i}\left(\Phi^{n}\left(t, \xi^{n}(\omega)\right)\right)=-J\left(\Phi_{i}^{n}\left(t, \xi^{n}(\omega)\right)-c_{i}^{\left.\sigma_{\Phi^{n}\left(t, \xi^{n}\right.}(\omega)\right)}\right)
$$

Using (45), we conclude that

$$
\begin{aligned}
\|V\|_{L^{2}\left(\mu_{t}^{n, \omega}\right)}^{2}=\int_{\mathbb{R}^{2 d}}\left|V_{t}\right|^{2} d \mu_{t}^{n, \omega} & =\frac{1}{n} \sum_{i=1}^{n}\left|\Phi_{i}^{n}\left(t, \xi^{n}(\omega)\right)-c_{i}^{\sigma_{\Phi^{n}\left(t, \xi^{n}(\omega)\right)}}\right|^{2} \\
& \leq \frac{8}{n} \sum_{i=1}^{n}\left(\left|\xi_{i}(\omega)\right|^{2}+(T+1)^{2} E^{2}\right. \\
& \leq 8\left(W_{2}^{2}\left(\mu_{o}^{n, \omega}, \delta_{0}\right)+(T+1)^{2} E^{2}\right) \\
& \leq a^{2}(\omega) .
\end{aligned}
$$

By (39), and the definition of $\mu_{t}^{n, \omega}$, we have

$$
\frac{d}{d t} \mu_{t}^{n, \omega}+\nabla \cdot\left(\mu_{t}^{n, \omega} V_{t}\right)=0
$$

in the distributional sense on $\mathbb{R}^{2 d}$. Using theorem 8.3 .1 in [2] and combining (47), (48), we obtain for $0 \leq s<t \leq T$ that

$$
W_{2}\left(\mu_{t}^{n, \omega}, \mu_{s}^{n, \omega}\right) \leq \int_{s}^{t}\left\|V_{l}\right\|_{L^{2}\left(\mu_{l}^{n, \omega}\right)} d l \leq(t-s) a(\omega),
$$

which proves (ii).

Lemma 3. For each $\omega \in \Omega^{\prime}$ ( $\Omega^{\prime}$ being defined right before (42)), there exists a collection $\left\{\mu_{t}^{\omega}\right\}_{t \in[0, T], \omega \in \Omega^{\prime}}$ and a subsequence $\left\{\mu_{t}^{n_{k}, \omega}\right\}_{k=1}^{\infty}$ of $\left\{\mu_{t}^{n, \omega}\right\}_{n=1}^{\infty}$ (depending on $\omega$ but independent of $t$ ) such that

(i)

$$
\lim _{k \rightarrow+\infty} W_{2}\left(\mu_{t}^{n_{k}, \omega}, \mu_{t}^{\omega}\right)=0 .
$$

(ii) We have $\mu_{o}^{\omega}=\mu_{o}$ and for $s, t \in[0, T]$,

$$
W_{2}\left(\mu_{t}^{\omega}, \mu_{s}^{\omega}\right) \leq|t-s| a(\omega) .
$$


Proof. First, by (37), (i) holds for $t=0$ and so, $\mu_{o}^{\omega}=\mu_{o}$ for $\mathbb{P}$-almost every $\omega \in \Omega$ which proves the first statement in (ii). Let $\omega \in \Omega^{\prime}$. We use (45) and the convex increasing function in (36) to conclude that

$$
\begin{aligned}
\int_{\mathcal{R}^{2 d}} \zeta\left(|z|^{2} / 4\right) d \mu_{t}^{n, \omega}(z) & =\frac{1}{n} \sum_{i=1}^{n} \zeta\left(\left|\phi_{i}^{n}\left(t, \xi^{n}(\omega)\right)\right|^{2} / 4\right) \\
& \leq \frac{1}{n} \sum_{i=1}^{n} \zeta\left(\left(\left|\xi_{i}(\omega)\right|+E T\right)^{2} / 4\right) \\
& \leq \frac{1}{n} \sum_{i=1}^{n} \zeta\left(\left(\left|\xi_{i}(\omega)\right|^{2}+E^{2} T^{2}\right) / 2\right) \\
& \leq \frac{1}{2 n} \sum_{i=1}^{n} \zeta\left(\left|\xi_{i}(\omega)\right|^{2}\right)+\zeta\left(T^{2} E^{2}\right) \\
& \leq \frac{1}{2} a_{\zeta}(\omega) \leq a_{\zeta}(\omega) .
\end{aligned}
$$

Above, we have used the convexity and the monotonicity of $\zeta$, and have set

$$
a_{\zeta}(\omega)=\sup _{n \geq 1}\left(\frac{1}{n} \sum_{i=1}^{n} \zeta\left(\left|\xi_{i}(\omega)\right|^{2}\right)+\zeta\left(T^{2} E^{2}\right)\right) .
$$

Since $\omega \in \Omega^{\prime}$, we have that $a_{\zeta}(\omega)$ is finite. Let

$$
\mathcal{K}(\omega)=\left\{\mu \in \mathcal{P}_{2}\left(\mathbb{R}^{2 d}\right): \int_{\mathbb{R}^{2 d}} \zeta\left(|z|^{2} / 4\right) d \mu(z) \leq a_{\zeta}^{2}(\omega)\right\} .
$$

Then $\mathcal{K}(\omega)$ is a compact subset of $\left(\mathcal{P}_{2}\left(\mathbb{R}^{2 d}\right), W_{2}\right)$ Indeed, (50) and the Banach-Alaoglu theorem ensures that every sequence of $\mathcal{P}_{2}\left(\mathbb{R}^{2 d}\right)$ admits a narrowly convergent subsequence, whose second moments converge to the second moments of its limit. By (50), $t \rightarrow \mu_{t}^{n, \omega}$ is a path in $\mathcal{K}(\omega)$ and remark 3 (ii) gives that the collection of the paths is uniformly Lipschitz. Consequently, there exists a subsequence $\left\{\mu_{t}^{n_{k}, \omega}\right\}_{k=1}^{\infty}$ of $\left\{\mu_{t}^{n, \omega}\right\}_{n=1}^{\infty}$ (depending on $\omega$ but independent of $t$ ) such that (49) holds. This proves (i). Now, (ii) is a direct consequence of (i) and (46).

Suppose in the remaining of this section that $\left\{\nu_{n}\right\}_{n=1}^{\infty}$ converges to $\nu$ in the sense of the Wasserstein distance, where we recall that

$$
\nu_{n}=1 / n \sum_{i=1}^{n} \delta_{c_{i}^{n}} .
$$

Define the Hamiltonian $H: \mathcal{P}_{2}\left(\mathbb{R}^{2 d}\right) \rightarrow \mathbb{R}$ by

$$
H[\mu]=1 / 2 W_{2}^{2}(\mu, \nu) .
$$

We next obtain a transport equation without imposing a severe restriction on $\mu_{o}$. 
Theorem 3 (Transport equation for $\left.\mu_{t}^{\omega}\right)$. Assume that $(\mathrm{C} 1)$ and $(\mathrm{C} 2)$ hold. Let $\left\{\mu_{t}^{\omega}: t \in[0, T], \omega \in \Omega^{\prime}\right\}$ be the collection of probability measures of Lemma 3 and suppose that $\nu$ vanishes on $(2 d-1)$-rectifiable sets so that $\Gamma_{o}\left(\mu_{t}^{\omega}, \nu\right)$ contains only one element (see [5] or [18] ). Then, there exists a collection $\left\{\gamma_{t}^{\omega}: t \in[0, T], \omega \in \Omega^{\prime}\right\}$ of probability measures on $\mathbb{R}^{2 d} \times \mathbb{R}^{2 d}$ which satisfies the following:

(i) $\gamma_{t}^{\omega}$ has $\mu_{t}^{\omega}$ and $\nu$ as first and second marginals and its support is cyclically monotone.

(ii) recalling that $z=(x, v)$ and $c=(a, b)$, we have for $g \in C_{c}^{1}\left(\mathbb{R}^{2 d}\right)$,

$$
\frac{d}{d t} \int_{\mathbb{R}^{2 d}} g d \mu_{t}^{\omega}=\int_{\mathbb{R}^{2 d} \times \mathbb{R}^{2 d}}\left(\left\langle\nabla_{x} g(z) ; v-b\right\rangle-\left\langle\nabla_{v} g(z) ; x-a\right\rangle\right) d \gamma_{t}^{\omega}(z ; c),
$$

in the sense of distributions on $(0, T)$.

(iii) We have $\mu_{o}^{\omega}=\mu_{o}$ and for $s, t \in[0, T]$,

$$
W_{2}\left(\mu_{t}^{\omega}, \mu_{s}^{\omega}\right) \leq|t-s| a(\omega) .
$$

(iv) We have $H\left[\mu_{t}^{\omega}\right]=H\left[\mu_{o}\right]$ for all $t \in[0, T]$.

Proof. Lemma 3 and the fact that $\omega \in \Omega^{\prime}$ give (iii).

For $\omega \in \Omega^{\prime}$, we define a probability measure $\gamma_{t}^{n, \omega}$ on $\mathbb{R}^{2 d} \times \mathbb{R}^{2 d}$ which has $\mu_{t}^{n, \omega}$ and $\nu^{n}$ as its marginals and whose support is cyclically monotone. Since $\omega \in \Omega^{\prime}, H^{n}$ is differentiable at $\xi^{n}(\omega)=\left(\xi_{1}(\omega), \cdots, \xi_{n}(\omega)\right)$ and so $\gamma_{t}^{n, \omega}$ is uniquely determined for $\mathcal{L}^{1}$-almost every $t \in(0, T)$. We have

$$
\int_{\mathbb{R}^{2 d} \times \mathbb{R}^{2 d}} F d \gamma_{t}^{n, \omega}=\frac{1}{n} \sum_{i=1}^{n} F\left(\Phi_{i}^{n}\left(t, \xi^{n}(\omega)\right), c_{i}^{\sigma_{\Phi^{n}\left(t, \xi^{n}(\omega)\right)}}\right) .
$$

We use (48) to obtain that, for $g \in C_{c}^{1}\left(\mathbb{R}^{2 d}\right)$,

$$
\begin{aligned}
\frac{d}{d t} & \int_{\mathbb{R}^{2 d}} g d \mu_{t}^{n, \omega}=\int_{\mathbb{R}^{2 d}}\left\langle\nabla g(z) ; V_{t}(z)\right\rangle d \mu_{t}^{n, \omega}(z) \\
& =\frac{1}{n} \sum_{i=1}^{n}\left\langle\nabla g\left(\Phi_{i}^{n}\left(t, \xi^{n}(\omega)\right)\right) ; V_{t}\left(\Phi_{i}^{n}\left(t, \xi^{n}(\omega)\right)\right)\right\rangle \\
& =-\frac{1}{n} \sum_{i=1}^{n}\left\langle\nabla g\left(\Phi_{i}^{n}\left(t, \xi^{n}(\omega)\right)\right) ; J\left(\Phi_{i}^{n}\left(t, \xi^{n}(\omega)\right)-c_{i}^{\left.\sigma_{\Phi^{n}\left(t, \xi^{n}\right.}(\omega)\right)}\right)\right\rangle \\
& =\int_{\mathbb{R}^{2 d} \times \mathbb{R}^{2 d}}\left(\left\langle\nabla_{x} g(z) ; v-b\right\rangle-\left\langle\nabla_{v} g(z) ; x-a\right\rangle\right) d \gamma_{t}^{n, \omega} .
\end{aligned}
$$

Lemma 3 ensures existence of a subsequence $\left\{\mu_{t}^{n_{k}, \omega}\right\}_{k=1}^{\infty}$ of $\left\{\mu_{t}^{n, \omega}\right\}_{n=1}^{\infty}$ which converges to $\mu_{t}^{\omega}$ in the $W_{2}$ metric. Since $\left\{\nu^{n_{k}}\right\}_{k=1}^{\infty}$ converges to $\nu$ in the $W_{2}$ metric, we conclude that $\left\{\gamma^{n_{k}, \omega}\right\}_{k=1}^{\infty}$ converges narrowly to the unique 
element of $\gamma_{t}^{\omega} \in \Gamma_{o}\left(\mu_{t}^{\omega}, \nu\right)$. Also, the second moments of $\left\{\gamma^{n_{k}, \omega}\right\}_{k=1}^{\infty}$ converge to the second moments of $\gamma_{t}^{\omega}$. This, together with (52), yields (i) and (ii).

$$
\begin{aligned}
2 H\left[\mu_{t}^{\omega}\right] & =W_{2}^{2}\left(\mu_{t}^{\omega}, \nu\right)=\lim _{k \rightarrow+\infty} W_{2}^{2}\left(\mu_{t}^{n_{k}, \omega}, \nu^{n_{k}}\right) \\
& =2 \lim _{k \rightarrow+\infty} H^{n_{k}}\left[\Phi^{n_{k}}\left(t, \xi^{n_{k}}(\omega)\right]=2 \lim _{k \rightarrow+\infty} H^{n_{k}}\left[\xi^{n_{k}}(\omega)\right]\right. \\
& =\lim _{k \rightarrow+\infty} W_{2}^{2}\left(\mu_{o}^{n_{k}, \omega}, \nu^{n_{k}}\right)=W_{2}^{2}\left(\mu_{o}^{\omega}, \nu\right)=2 H\left[\mu_{o}\right] .
\end{aligned}
$$

We have used proposition 1 to obtain the second inequality in (53).

The second main theorem of this section states a transport equation for the measures $\left\{\mu_{t}^{\omega}\right\}$. It asserts that these measures are absolutely continuous with respect to Lebesgue measure on $\mathbb{R}^{2 d}$. In particular, when $d=1$, it proves that the empirical measures in (44) converge to absolutely continuous measures which satisfy the semigeostrophic system.

Theorem 4 (Transport equation with absolutely continuous measures). Assume that (H1) and (H2) hold. Let $\left\{\mu_{t}^{\omega}\right\}_{t \in[0, T], \omega \in \Omega^{\prime}}$ be the collection of probability measures of Lemma 3, obtained as points of accumulation for the $W_{2}$-distance, of the empirical measures in (44). Suppose that $\left\{\nu^{n}\right\}_{n=1}^{\infty}=\left\{1 / n \sum_{i=1}^{n} \delta_{c}^{i}\right\}_{n=1}^{\infty}$ converges in the metric $W_{2}$ to a measure $\nu$ whose support is compact. Then there exists a $\mathbb{P}$-measurable set $\Omega^{\prime \prime} \subset \Omega^{\prime}$ such that $\mathbb{P}\left[\Omega^{\prime \prime}\right]=1$ and the following hold for $\omega \in \Omega^{\prime \prime}$ :

(i) (absolute continuity) $\mu_{t}^{\omega}<<\mathcal{L}^{2 d}$ and there exists convex, uniformly Lipschitz functions $\phi_{t}^{\omega}: \mathbb{R}^{2 d} \rightarrow \mathbb{R}$ such that $\left(\nabla \phi_{t}^{\omega}\right)_{\#} \mu_{t}^{\omega}=\nu$.

(ii) (transport equation)

$$
\frac{d}{d t} \mu_{t}^{\omega}+\operatorname{div}_{x}\left(\left(v-\nabla_{v} \phi_{t}^{\omega}\right) \mu_{t}^{\omega}\right)=\operatorname{div}_{v}\left(\left(x-\nabla_{x} \phi_{t}^{\omega}\right) \mu_{t}^{\omega}\right)
$$

in the sense of distributions.

(iii) $\mu_{0}^{\omega}=\mu_{o}$ and, for all $s, t \in[0, T]$,

$$
W_{2}\left(\mu_{t}^{\omega}, \mu_{s}^{\omega}\right) \leq|t-s| a(\omega) .
$$

(iv) (conservation of the Hamiltonian) We have $H\left[\mu_{t}^{\omega}\right]=H\left[\mu_{o}\right]$ for $t \in[0, T]$.

Proof. As in the proof of theorem 3, there exists a subsequence $\left\{\mu_{t}^{n_{k}, \omega}\right\}_{k=1}^{\infty}$ of $\left\{\mu_{t}^{n, \omega}\right\}_{n=1}^{\infty}$ which converges to $\mu_{t}^{\omega}$ in the $W_{2}$ metric. Lemma 3 , and the fact that $\omega \in \Omega^{\prime}$, give (iii).

For $\omega \in \Omega, n$ an integer and $t \in[0, T], \Phi_{t}^{n, \omega}: \mathbb{R}^{2 n d} \rightarrow \mathbb{R}^{2 n d}$ preserves the $\mathcal{L}^{2 n d}$ Lebesgue measure. By remark 3, there exists a constant $R_{1}>1 / 2$ such that

$$
\left|\left(\Phi_{t}^{n, \omega}(\mathbf{z})\right)_{i}\right| \leq R_{1} \quad z_{1}, \cdots, z_{n} \in B_{R_{o}} .
$$

By theorem 2 for $p \in(0,1)$ and $A \subset \mathbb{R}^{2 d}$ a Borel set such that $2 C_{o}\left|Q_{R_{1}}\right||A|^{p}<$ 1 , we have

$$
\mathbb{P}\left\{\omega \in \Omega: \mu_{t}^{\omega}[A]>p\right\}=0
$$


for each $t \in[0, T]$. We set $C=2 C_{o}\left|Q_{R_{1}}\right|$ and use theorem 2 to obtain the existence of a $\mathbb{P}$-measurable set $\Omega_{t}^{\prime \prime} \subset \Omega^{\prime}$ such that $\mathbb{P}\left[\Omega_{t}^{\prime \prime}\right]=1$ and

$$
\mu_{t}^{\omega}[A] \leq \frac{\ln (2 C)}{-\ln |A|}
$$

for all $\omega \in \Omega_{t}^{\prime \prime}$, all $A \subset \mathbb{R}^{2 d}$ such that $2 C|A|<1$ and all $t \in[0, T] \cap \mathbb{Q}$. In fact these assertions hold for all $t \in[0, T]$. We set

$$
\Omega^{\prime \prime}=\cap_{t \in[0, T] \cap \mathbb{Q}} \Omega_{t}^{\prime \prime}
$$

and obtain that $\mathbb{P}\left[\Omega^{\prime \prime}\right]=1$. Recall that (iii) gives us that the path $t \rightarrow \mu_{t}^{\omega}$ is a Lipschitz path. We use this, together with the fact that (54) holds for open sets and $t$ rational, to extend (54) to all $t \in[0, T]$ all $\omega \in \Omega^{\prime \prime}$ and all open sets $A \subset \mathbb{R}^{2 d}$ such that $2 C|A|<1$. Consequently, we have proven that $\mu_{t}^{\omega}<<\mathcal{L}^{2 d}$ for all $t \in[0, T]$ all $\omega \in \Omega^{\prime \prime}$. The Monge-Kantorovich theory ensures that $\Gamma_{o}\left(\mu_{t}^{\omega}, \nu\right)$ has exactly one element (see [5] or [18] ) and so, the sequence $\left\{\gamma^{n_{k}, \omega}\right\}_{k=1}^{\infty}$ of theorem 3 converges narrowly to the unique element $\gamma_{t}^{\omega} \in \Gamma_{o}\left(\mu_{t}^{\omega}, \nu\right)$. Also, we have existence of a convex function $\phi_{t}^{\omega}: \mathbb{R}^{2 d} \rightarrow \mathbb{R}$ such that

$$
\partial \phi_{t}^{\omega} \subset \mathbb{R}^{2 d} \times \operatorname{spt} \nu, \quad\left(\nabla \phi_{t}^{\omega}\right)_{\#} \mu_{t}^{\omega}=\nu, \quad \gamma_{t}^{\omega}=\left(\mathbf{i d} \times \nabla \phi_{t}^{\omega}\right)_{\#} \mu_{t}^{\omega} .
$$

We have completed the proof of (i). These, together with (51 ) yields (ii). The proof of (iv) is similar to that of theorem 3 (iv).

Remark 4. (i) When $d=1$ and $\nu=\chi_{Q} \mathcal{L}^{2}$ for $Q \subset \mathbb{R}^{2}$, then the transport equation in theorem 4 (ii) is nothing but the semigeostrophic equations.

(ii) As a corollary of theorem 4, we have the following: assume that $\mu_{o}$ is a probability density on $\mathbb{R}^{2}$, of compact support, with an $L^{\infty}$ density. Let $\mu_{o}^{n}=1 / n \sum_{i=1}^{n} \delta_{\left(x_{i}^{n}(0), v_{i}^{n}(0)\right)}$ be a sequence of discrete measures converging to $\mu_{o}$ in the $W_{2}$-distance. Fix $T>0$ and let $t \rightarrow\left(x_{i}^{n}(t), v_{i}^{n}(t)\right)$ be a solution in $[0, T]$ of the differential equation in (2) with $\left(x_{i}^{n}(0), v_{i}^{n}(0)\right)$ as initial values. Set

$$
\mu_{t}^{n}=1 / n \sum_{i=1}^{n} \delta_{\left(x_{i}^{n}(t), v_{i}^{n}(t)\right)} .
$$

Then, we can always choose the initial values $\left(x_{i}^{n}(0), v_{i}^{n}(0)\right)$ appropriately so that

- (2) has a solution.

- There exists an increasing sequence $\left\{n_{k}\right\}_{k=1}^{\infty}$ such that for $t \in[0, T]$, $\left\{\mu_{t}^{n_{k}}\right\}$ tends to $\mu_{t}$ as $k$ tends to $+\infty$.

- $\mu_{t}<<\mathcal{L}^{2}$ and satisfies the semigeostrophic system.

Corollary 1 (Vlasov-Monge-Ampère system). Assume that (H1) and (H2) hold and that $\nu=\left(\chi_{Q_{1}} \mathcal{L}^{d}\right) \times \delta_{0}$, where $Q_{1}=(-1 / 2,1 / 2)^{d}$. Let $\mu_{t}^{\omega}=$ $f_{t} \mathcal{L}^{2 d}$, let $\phi_{t}^{\omega}$ be as in Theorem 4 and define $\rho_{t}(x)=\int_{\mathbb{R}^{d}} f_{t}(x, v) d v$. Then, 
There exists $\Phi_{t} \equiv \Phi_{\rho_{t}}$ such that $\left(\mathbf{i d}-\nabla_{x} \Phi_{\rho_{t}}\right) \# \rho_{t} \mathcal{H}^{d}=\chi_{Q_{1}} \mathcal{H}^{d}, \quad|x|^{2} / 2-$ $\Phi_{\rho_{t}}(x)$ is a convex function in the $x$ variables and

$$
\begin{cases}\partial_{t} f(t, x, v)+\operatorname{div}_{x}(v f(t, x, v)) & =\operatorname{div}_{v}\left(f(t, x, v)\left(\nabla_{x} \Phi_{\rho_{t}}(x)\right)\right. \\ \operatorname{det}\left(I_{d}-\nabla_{x x}^{2} \Phi_{\rho_{t}}(x)\right) & =\rho_{t}(x) .\end{cases}
$$

Proof. Let $\omega \in \Omega^{\prime \prime}$. As argued in (14), there exists a convex function $\varphi_{t}^{\omega}: \mathbb{R}^{d} \rightarrow \mathbb{R}$ such that $\phi_{t}^{\omega}(x, v)=\varphi(x)$ and $(\nabla \varphi)_{\#} \rho_{t} \mathcal{L}^{d}=\chi_{Q_{1}} \mathcal{L}^{d}$. We use theorem 4 (ii) to conclude that (55) holds.

Acknowledgements. It is a pleasure to express our gratitude to M. Loss for the many discussions we had and for his continuing support. We received crucial imputs from L. Ambrosio and H. Matzinger on section 2. Fruitful discussions and criticisms were also provided by Y. Brenier, E. Carlen, V. Koltchinskii and T. Nguyen. WG gratefully acknowledges the support provided by NSF grants DMS-02-00267, DMS-03-754729 and the hospitality of MSRI, Berkeley, CA. The postdoctoral fellowship of GP was partially supported by the FRG grant DMS03-754729. MC was partially supported by the FRG grant DMS-03-754729.

\section{References}

1. L. Ambrosio. Transport equation and Cauchy problem for BV vector fields. Invent. Math., 158, 227-260 (2004).

2. L. Ambrosio, N. Gigli and G. Savaré. Gradient flows in metric spaces and the Wasserstein spaces of probability measures. Lectures in Mathematics ETH Zürich, Birkhäuser Verlag, 2005.

3. H. Aref. Integrable, chaotic, and turbulent vortex motion in two-dimensional flows. Ann. Rev. Fluid Mech. 15, 345 (1983).

4. J. Batt and G. Rein. Global classical solutions of the periodic Vlasov-Poisson system. C.R. Acad. Sci. Paris Sér. I Math., 313: 411-416, (1991).

5. Y. Brenier. Décomposition polaire et réarrangement monotone des champs de vecteurs. C.R. Acad. Sci. Paris Sér. I Math., 305:805-808, (1987).

6. Y. Brenier. A geometric presentation of the semi-geostrophic equations. Unpublished Notes. Newton Institute.

7. Y. Brenier. Derivation of the Euler equations from a caricature of Coulomb interaction. Comm. Math. Physics, 212, 93-104 (2000).

8. Y. Brenier. Convergence of the Vlasov-Poisson system to the incompressible Euler equations. Comm. Partial Differential Equations, 25, 737-754 (2000).

9. J.D. Benamou and Y. Brenier. Weak existence for the semigeostrophic equations formulated as a coupled Monge-Ampère equations/transport problem. SIAM J. Appl. Ana. Math. 58, no 5, 1450-1461 (1998).

10. Y. Brenier and G. Loeper. A geometric approximation to the Euler equations: The Vlasov-Monge-Ampere equation. Geom. Funct. Anal. 14, 11821218 (2004).

11. E. Carlen and W. Gangbo. Constrained steepest descent in the 2-Wasserstein metric. Annals of Mathematics 157 , 807-846 (2003).

12. M.J.P. Cullen. A mathematical theory of large-scale atmospheric flow. Imperial Colege Press, 259pp. (2006). 
13. M.J.P. Cullen and W. Gangbo. A variational approach for the 2-dimensional semi-geostrophic shallow water equations. Arch. Ration. Mech. Anal. 156 241-273 (2001).

14. M.J.P. Cullen and R.J. Purser. An extended lagrangian theory of semigeostrophic frontogenesis. J. Atmosph. Sciences 41, 1477-1497 (1984).

15. M.J.P. Cullen and R.J. Purser. Properties of the lagrangian semigeostrophic equations. J. Atmosph. Sciences vol 40, 17, 2684-2697 (1989).

16. A Eliassen. The quasi-static equations of motion. Geofys. Publ., 17, No 3, (1948).

17. L.C. Evans. Partial differential equations and Monge-Kantorovich mass transfer. Int. Press, Current Dev. Math, 26, 26-78, 1997.

18. W. Gangbo and R.J. McCann. The geometry of optimal transportation. Acta Math. 177 113-161 (1996).

19. B.J. Hoskins. The geostrophic momentum approximation and the semigeostrophic equations. J. Atmosph. Sciences 32, 233-242, (1975).

20. G. Loeper. Quasi-neutral limit of the Euler-Poisson and Euler-Monge-Ampere systems. To appear in Comm. Partial Differential Equations 30 issue 8, 11411167 (2005).

21. P.-L. Lions and B. Perthame. Propagation of moments and regularity for the 3-dimensional Vlasov-Poisson system. Invent. Math. 105, 415-430, (1991).

22. K. Pfaffelmoser. Global classical solutions of the Vlasov-Poisson system in three dimensions for general initial data. Jour. Differential Equations 95, 281-303, (1992).

23. I. Roulstone and J. Norbury. A Hamiltonian structure with contact geometry for the semi-geostrophic equations. J. Fluid Mech. 272, 211-233, (1994)

\author{
M.Cullen \\ Met Office \\ Exeter, United Kingdom \\ e-mail: mike.cullen@.metoffice.gov.uk \\ and \\ W. Gangbo \& G. Pisante \\ School of Mathematics, Georgia Institute of Technology \\ Atlanta, GA 30332, USA \\ e-mail: gangbo@math.gatech.edu
}

